\title{
MicroRNA-211 suppresses prostate cancer proliferation by targeting SPARC
}

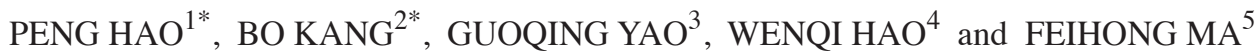 \\ ${ }^{1}$ Department of Urology, First Affiliated Hospital of Jiamusi University, Jiamusi, Heilongjiang 154003; \\ ${ }^{2}$ Central Sterile Supply Department, The First Affiliated Hospital of Harbin Medical University, Harbin, Heilongjiang 150001; \\ ${ }^{3}$ Department of Surgery 1, The 224th Hospital of Chinese People's Liberation Army, Jiamusi, Heilongjiang 154002; \\ ${ }^{4}$ Department of Clinical Medicine, School of Clinical Medicine, Jiamusi University; ${ }^{5}$ Department of Interventional Radiology, \\ First Affiliated Hospital of Jiamusi University, Jiamusi, Heilongjiang 154003, P.R. China
}

Received February 23, 2017; Accepted November 20, 2017

DOI: $10.3892 / 01.2018 .7877$

\begin{abstract}
Dysregulation of microRNAs (miRNAs/miRs) is frequently associated with cancer progression. Altered expression of miR-211 has been observed in various types of human cancer; however, its expression and role in prostate cancer (PCa) remains unknown. In the present study, the expression of miR-211 in PCa cell lines and tissues was measured by reverse transcription-quantitative PCR (qPCR), revealing that miR-211 was downregulated in PCa cell lines and tissues. Further analysis revealed that low miR-211 was associated with the tumor stage and Gleason score. With the assistance of miR-211 mimics and inhibitor, it was also revealed that the overexpression of miR-211 could inhibit PCa cell proliferation in vitro. Conversely, downregulated miR-211 expression promotes PCa cell proliferation. In addition, the secreted protein acidic and rich in cysteine (SPARC) was identified as a target of miR-211 in the PCa cell lines, and SPARC expression was inversely associated with miR-211. In conclusion, it was demonstrated that the miR-211 expression was downregulated in PCa cell lines and tissues. Additionally, miR-211 could inhibit PCa cell proliferation partially by downregulating SPARC. Therefore, miR-211 may be a potential therapeutic target for $\mathrm{PCa}$ treatment in the future.
\end{abstract}

\section{Introduction}

Prostate cancer (PCa) is the most prevalent type of cancer among men in the United States (1) and currently accounts

Correspondence to: Dr Feihong Ma, Department of Interventional Radiology, First Affiliated Hospital of Jiamusi University, 348 Dexiang Street, Jiamusi, Heilongjiang 154003, P.R. China

E-mail: feihong_ma@aliyun.com

${ }^{*}$ Contributed equally

Key words: microRNA-211, secreted protein acidic and rich in cysteine, prostate cancer, proliferation for $30 \%$ of all diagnosed cancers (2). PCa is the second most common cause of cancer-associated mortality in men in the United States (2). A recent study estimated that 180,890 men in the United States were diagnosed with $\mathrm{PCa}$ and 26,120 patients in the United States succumbed to PCa in 2016 (2). In China, the incidence of $\mathrm{PCa}$ is also increasing $(3,4)$. No standard methods for PCa prevention, early diagnosis, treatment or prognosis are currently available (5). Therefore, the majority of patients with $\mathrm{PCa}$ are diagnosed at the late stage, leading to high mortality rates (3). Identifying novel diagnostic and prognostic markers that benefit the early diagnosis and treatment of $\mathrm{PCa}$ are required to improve the treatment of the disease.

MicroRNAs (miRNAs/miRs) are endogenous, small non-coding RNAs that are 22 nucleotides in length (6) and were initially discovered as a small temporal RNA in Caenorhabditis elegans in 1993 (7). The functions of miRNA have been revealed to serve a notable role in various biological processes, including cell differentiation (8), proliferation $(8,9)$, apoptosis (10) and development (11). To date, $>2,500$ potential human miRNAs are identified and recorded in the miRBase, and $>30 \%$ of all genes are estimated to be regulated by miRNAs (12). In recent years, mounting evidence has demonstrated that miR-211 can impact cell proliferation and migration in numerous human cancers (13-18). However, the role of miR-211 in tumor progression remains uncertain. In non-small lung cancer, Ye et al (18) revealed the miR-211 can directly downregulate the expression of SRC kinase signaling inhibitor 1 (SRCIN1), and promote non-small cell lung cancer proliferation. As a comparison, miR-211 expression was downregulated in hepatocellular carcinoma $(15,16)$, gastric cancer (14) and epithelial ovarian cancer (17), and was regarded as a tumor suppressor by targeting the expression of its downstream targets. However, the expression and role of miR-211 in PCa remains unclear.

Secreted protein acidic and rich in cysteine (SPARC), also known as osteonectin, is a matricellular glycoprotein that serves instrumental roles during cell proliferation, migration and cell differentiation $(19,20)$. SPARC was identified to be upregulated in various tumors, including PCa (21-23); high SPARC expression was also revealed to be associated with 
aggressive stages of melanoma (24). Additionally, the expression of SPARC could be regulated by miR-211 in hepatocellular carcinoma (16). Meanwhile, a recent study demonstrated that SPARC could mediate metastatic dormancy of PCa in the bone (25), which highlighted the significant role of SPARC in $\mathrm{PCa}$. However, whether or not miR-211 could regulate the expression of SPARC in PCa remains unreported. The present study aimed to determine the expression and function of miR-211 in PCa and investigate the molecular mechanism of miR-211 in the progression of PCa.

\section{Materials and methods}

Participants. The study was approved by the Ethics Committee of the First Affiliated Hospital of Jiamusi University (Jiamusi, China), and was performed in accordance with the Declaration of Helsinki. Written informed consent was obtained from all patients. Matched PCa and normal prostate tissues 36 pairs, 44-71 years old (mean age, 62) were obtained from patients who underwent radical prostatectomy between October 2010 and February 2012 at the First Affiliated Hospital of Jiamusi University (Jiamusi, China). None of the patients had received radiotherapy or chemotherapy before surgical resection. PCa stage was classified according to the seventh American Joint Committee on Cancer (AJCC) classification system (26). All samples were snap-frozen in liquid nitrogen immediately and stored at $-80^{\circ} \mathrm{C}$ following surgery until further use.

Cell lines and cell culture. Two human PCa cell lines, DU145 and PC-3, were purchased from the American Type Culture Collection (Manassas, VA, USA) and cultured in RPMI-1640 medium (Invitrogen; Thermo Fisher Scientific, Inc.) supplemented with $10 \%$ fetal bovine serum (Invitrogen; Thermo Fisher Scientific, Inc.), $100 \mu \mathrm{g} / \mathrm{ml}$ penicillin and $100 \mu \mathrm{g} / \mathrm{ml}$ streptomycin (Invitrogen; Thermo Fisher Scientific, Inc., Waltham, MA, USA). Normal human prostate epithelial cells (NHPE) were purchased from Lonza, Inc. (Allendale, NJ, USA) and cultured in prostate epithelial cell growth medium containing growth medium and supplements (cat no. CC-3166; Lonza, Inc.). All the cell lines were incubated in a humidified atmosphere of $5 \% \mathrm{CO}_{2}$ at $37^{\circ} \mathrm{C}$.

Cell transfection. The miR-211 mimic (5'-UUCCCUUUG UCAUCCUUCGCCU-3'), inhibitor (5'-AGGCGAAGGAUG ACAAAGGGAA-3') and miRNA negative control (5'-CAG UACUUUUGUGUAGUACAA-3') molecules were purchased from Guangzhou RiboBio Co., Ltd. (Guangzhou, China). The small interfering RNA (siRNA) against SPARC (5'-GCA GAGGUGACUGAGGUAUCU-3') (2.5 nmol) and negative control (5'-AGUCGAGAUCGGUGUUAGCAG-3') (2.5 nmol) were designed and synthesized by Shanghai GenePharma Co., Ltd. (Shanghai, China). These nucleotides (miRNAs or siRNAs) were transfected into the cell lines $\left(2 \times 10^{5}\right.$ cells/well, DU145 and PC-3) using Lipofectamine 2000 (Invitrogen; Thermo Fisher Scientific, Inc.) until a final concentration of $50 \mathrm{nM}$ and according to the manufacturer's protocol. At $48 \mathrm{~h}$ after transfection, cells were collected for reverse transcription-quantitative polymerase chain reaction (RT-qPCR) and western blot analyses.
Table I. Distribution of miR-211 expression status in human prostate cancer according to clinicopathological characteristics.

miR-211

expression, $\mathrm{n}$

\begin{tabular}{lrrrc} 
Characteristics & Patients, $\mathrm{n}$ & Low & High & P-value \\
\hline $\begin{array}{l}\text { Age, years } \\
\quad 60\end{array}$ & 26 & 21 & 5 & NS \\
$\quad<60$ & 10 & 8 & 2 & \\
Tumor stage & & & & 0.035 \\
pT2a-c & 18 & 12 & 6 & \\
pT3a-4 & 18 & 17 & 1 & \\
Gleason score & & & & 0.013 \\
$\geq 8$ & 28 & 25 & 3 & \\
$<8$ & 8 & 4 & 4 & \\
Nodal status & & & & NS \\
pN0 & 27 & 23 & 4 & \\
pN1 & 9 & 6 & 3 & \\
PSA levels, ng/ml & & & & NS \\
$\geq 10$ & 24 & 19 & 5 & \\
$<10$ & 12 & 10 & 2 & \\
\hline
\end{tabular}

NS, not significant; miR-211, microRNA-211; PSA, prostate specific antigen.

Total RNA isolation and RT-qPCR. Total RNA was extracted from cultured cells and from surgically resected fresh PCa tissues using TRIzol reagent (Beyotime Institute of Biotechnology, Haimen, China) according to the manufacturer's protocol. The RNA concentration was quantified using NanoDrop 2000 (Thermo Fisher Scientific, Inc.). To quantify the level of miR-211 expression, total RNA was polyadenylated and reverse transcribed using the TaqMan MicroRNA Reverse Transcription kit and TaqMan miRNA Assay kit (Applied Biosystems; Thermo Fisher Scientific, Inc.) according to the manufacturer's protocol. To quantify the level of SPARC expression, the SYBR Premix Ex TaqTM kit (Takara Biotechnology Co., Ltd., Dalian, China) was used. PCR conditions included an initial holding period at $95^{\circ} \mathrm{C}$ for $10 \mathrm{~min}$ for $1 \mathrm{cycle}$, then $95^{\circ} \mathrm{C}$ for $15 \mathrm{sec}$ and $60^{\circ} \mathrm{C}$ for $30 \mathrm{sec}$ for 40 cycles. Primers used in the present study were as follows: SPARC forward, 5'-AGCACCCCATTG ACGGGTA-3' and reverse, 5'-GGTCACAGGTCTCGA AAAAGC-3'; GAPDH forward, 5'-AAGGGAAGGTTG CTGGATAGG-3' and reverse, 5'-CACATCCACCTCCTC CACATC-3'. Each sample was repeated in triplicate. The relative expression of genes was calculated using the $2^{-\Delta \Delta \mathrm{Cq}}$ method (27).

Western blot analysis. Total proteins were extracted from surgically resected fresh $\mathrm{PCa}$ tissues using Radioimmunoprecipitation Assay lysis buffer (Beyotime Institute of Biotechnology). The protein concentration was quantified using a BCA protein concentration determination kit (Beyotime Institute of Biotechnology). An equal 


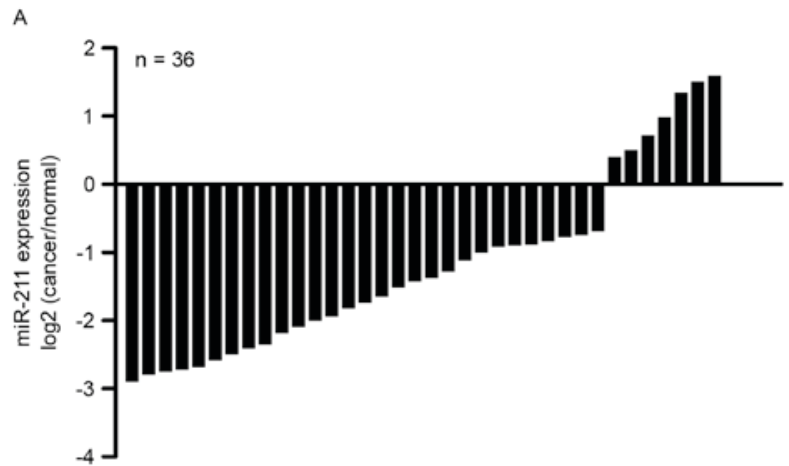

B

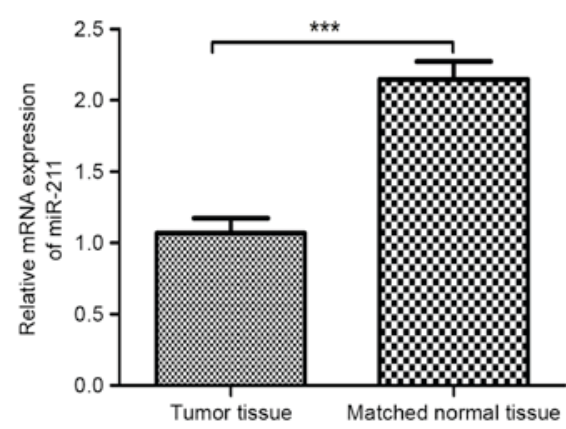

c

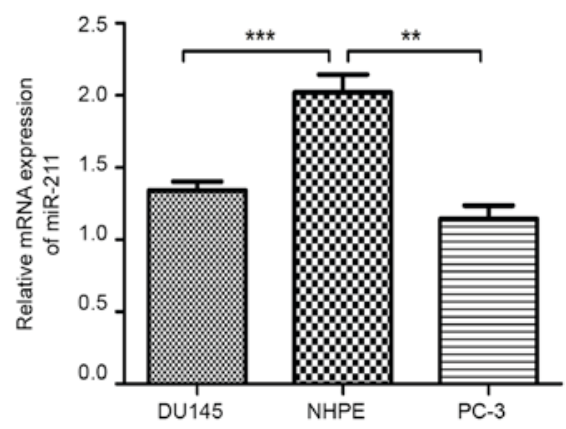

Figure 1. miR-211 expression is downregulated in PCa cell lines and tissues. (A) reverse transcription-quantitative polymerase chain reaction analysis of miR-211 expression in 36 pairs of PCa tissues and their matched normal prostate tissues. The expression of miR-211 was normalized to U6 small nuclear RNA. (B) The expression of miR-211 in PCa tissues was significantly lower than in adjacent tissues. (C) Expression levels of miR-211 in the DU145 and PC-3 PCa cell lines compared with NHPE cells. ${ }^{* *} \mathrm{P}<0.01$ and ${ }^{* * *} \mathrm{P}<0.001$. miR-211, microRNA-211; PCa, prostate cancer; NHPE, normal human prostate epithelium.

amount of protein samples $(50 \mu \mathrm{g})$ was separated using $10 \%$ SDS-PAGE. The separated protein was then transferred onto a polyvinylidene fluoride membrane (Beyotime Institute of Biotechnology), blocked in $5 \%$ fat-free milk at room temperature for $1 \mathrm{~h}$ and incubated with primary antibodies anti-SPARC (1:1,000; cat. no. ab207743; Abcam, Cambridge, MA, USA) and anti-GAPDH (1:1,000; cat. no. ab37168; Abcam) at $4^{\circ} \mathrm{C}$ overnight followed by incubation with a horseradish peroxidase-conjugated goat anti-rabbit secondary antibody (1:10,000; ab97080, Abcam) at room temperature for $1 \mathrm{~h}$. GAPDH was selected as an internal control. Results were detected using the BeyoECL Plus kit (Beyotime Institute of Biotechnology). The densitometry was analyzed using ImageJ 1.48 software (National Institutes of Health, Bethesda, MD, USA). Each experiment was repeated independently three times.

Cell proliferation assay. The cell proliferation rate assay was conducted using Cell Counting kit-8 (CCK-8; Beyotime Institute of Biotechnology) according to the manufacturer's protocol. In brief, the cell lines were seeded into a 96-well plate at a density of $2 \times 10^{3}$ cells/well and cultured in $100 \mu \mathrm{l}$ of the aforementioned medium. In total, $10 \mu \mathrm{l} \mathrm{CCK}-8$ reagent was added to each well at the indicated time points $(0,24$, 48 and $72 \mathrm{~h}$ ) after seeding and further incubated at the aforementioned conditions $\left(37^{\circ} \mathrm{C}\right)$ for $2 \mathrm{~h}$. The absorbance was measured at $450 \mathrm{~nm}$ using a microplate reader (Lifecare Medical Equipments Co., Ltd., Ningbo, Zhejiang, China). Cell medium without cells was treated with $10 \mu \mathrm{l}$ CCK-8 reagent at $0,24,48$ and $72 \mathrm{~h}$ and cultured for $2 \mathrm{~h}$ at $37^{\circ} \mathrm{C}$ then used as control to eliminate the background. Each experiment was performed in triplicate.

Bioinformatic analysis. The online algorithm TargetScan (http://www.targetscan.org/) (28) was used to predict the targets of miR-211. SPARC, whose expression was upregulated in PCa, was selected to further investigate whether it is regulated by miR-211.

Statistical analysis. The data are expressed as the mean \pm standard deviation of triplicate experiments. Data analysis was performed using the SPSS 17.0 software (SPSS, Inc., Chicago, IL, USA). Statistical analysis of differences between two groups was performed using Student's t-test; multiple comparisons were performed using one way analysis of variance with post hoc Tukey's test. Pearson's $\chi^{2}$ test was used for analyzing the association between the expression level of miR-211 and the clinicopathological features of patients. $\mathrm{P}<0.05$ was considered to indicate a statistically significant difference in all tests.

\section{Results}

miR-211 is downregulated in PCa tissues and cell lines. A decrease in miR-211 expression was identified in 29 of $36 \mathrm{PCa}$ tissues compared with the matched non-tumor tissues (Fig. 1A). As revealed in Fig. 1B, the expression of miR-211 in PCa tissues was significantly lower than that in the matched normal prostate tissues $(\mathrm{P}<0.001)$. The expression of miR-211 was also assessed in two PCa cell lines. As depicted in 


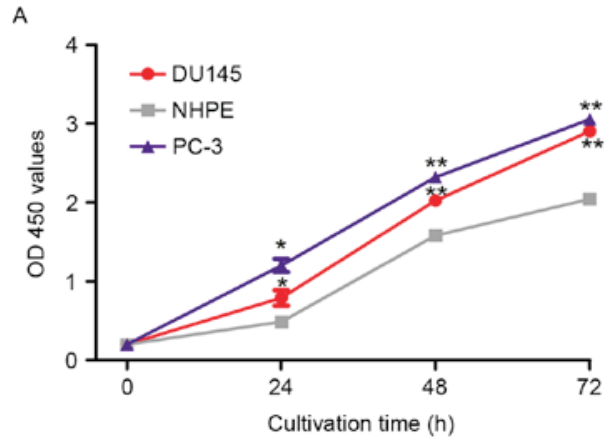

B
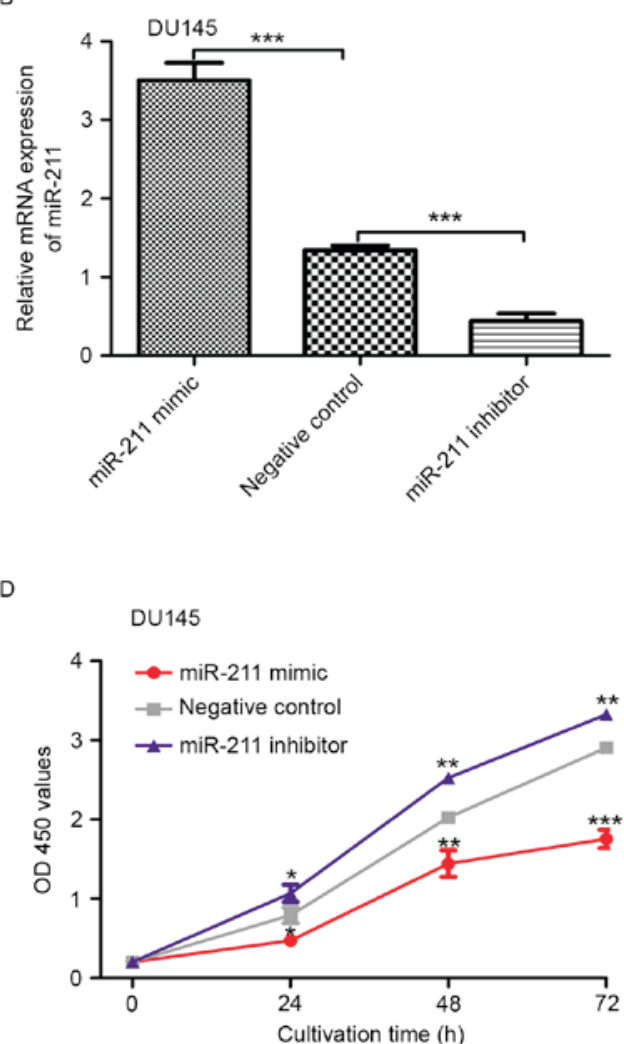

C

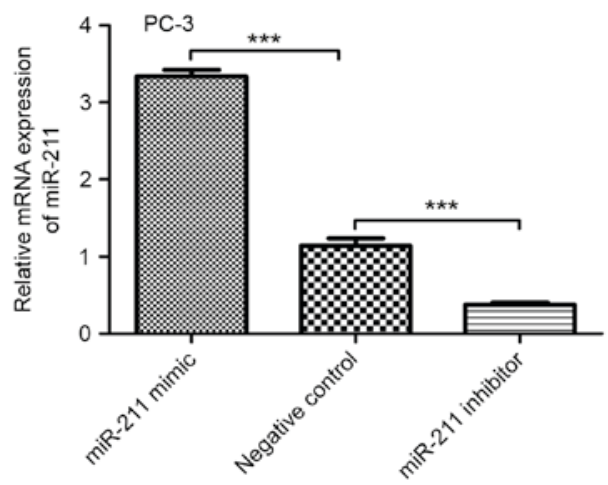

$\mathrm{E}$

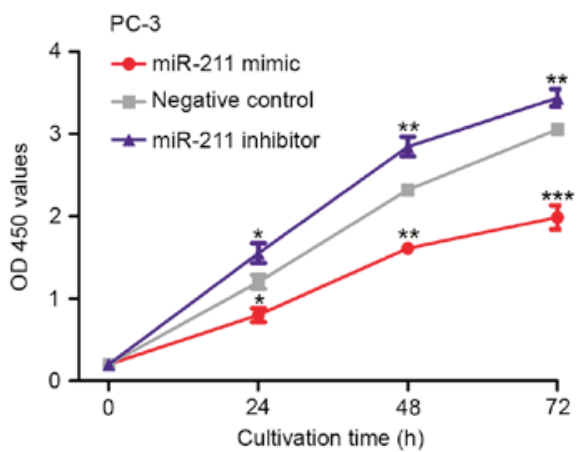

Figure 2. Upregulation of miR-211 inhibits cell proliferation. (A) CCK-8 assay revealed that the PCa cell lines DU145 and PC-3 had a significantly higher cell proliferation rate than NHPE cells. (B) Transfection with miR-211 mimics enhanced the expression of miR-211, whereas the miR-211 inhibitor repressed the expression of miR-211 in DU145 cells. (C) Transfection with miR-211 mimics enhanced the expression of miR-211 and miR-211 inhibitor can repress the expression of miR-211 in the PC-3 cells. (D) CCK-8 proliferation assay revealed that the overexpression of miR-211 significantly inhibited the growth rate of cells compared with that of the control in DU145 cells. Conversely, transfection with miR-211 inhibitor significantly promoted the proliferation of the DU145 cells. (E) CCK-8 proliferation assay revealed that the overexpression of miR-211 significantly inhibited the growth rate of cells compared with that of the control in PC-3 cells. Conversely, miR-211 inhibitor significantly promoted the proliferation of the PC- 3 cells. ${ }^{*} \mathrm{P}<0.05,{ }^{* * *} \mathrm{P}<0.01$ and ${ }^{* * *} \mathrm{P}<0.001$ vs. control. miR-211, microRNA-211; PCa, prostate cancer; CCK-8, Cell Counting kit-8; NHPE, normal human prostate epithelium.

Fig. 1C, the expression of miR-211 in the PCa DU145 and PC-3 cell lines was also evidently lower than in the NHPE cells $(\mathrm{P}<0.01)$.

miR-211 downregulation is associated with clinicopathological features in patients with PCa. To assess whether miR-211 has any prognostic significance, the association between miR-211 expression and clinicopathological features of patients with PCa was analyzed. As demonstrated in Table I, no statistical difference was revealed between miR-211 expression and age, nodal status and prostate specific antigen (PSA) levels (all $\mathrm{P}>0.05$ ). However, the expression of miR-211 was revealed to be closely associated with the tumor stage $(\mathrm{P}=0.035)$ and higher Gleason scores (29) $(\mathrm{P}=0.013)$.

Upregulation of miR-211 inhibits cell proliferation in vitro. To examine the functional significance of miR-211 in PCa, the rate of cell proliferation in PCa cell lines and NHPE cells was measured and compared. As depicted in Fig. 2A, the cell proliferation rate was evidently higher in the PCa cell lines than in NHPE cell line $(\mathrm{P}<0.05)$. The miR-211 mimic, inhibitor and corresponding negative control were selected to regulate the expression level of miR-211 in the PCa cell 
A

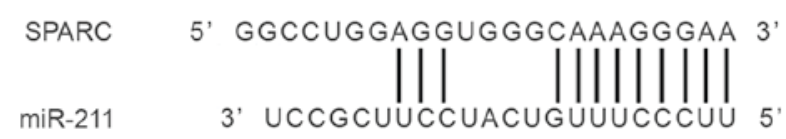

B

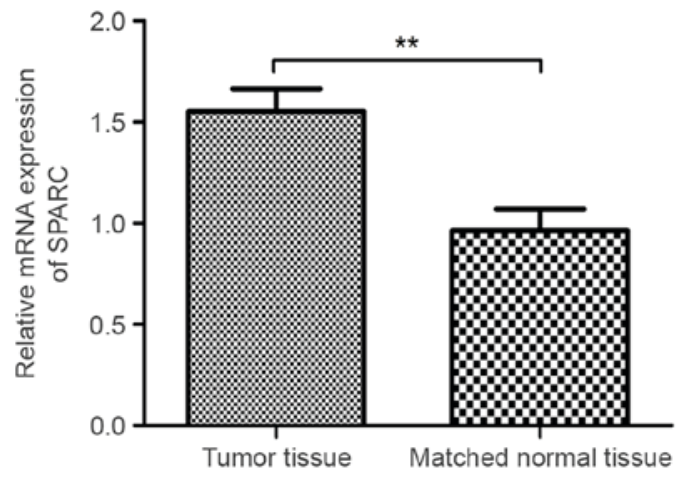

D

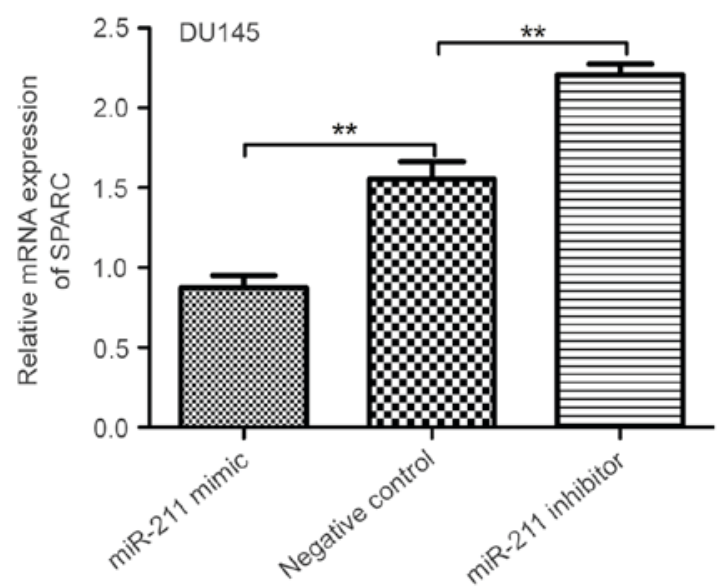

C
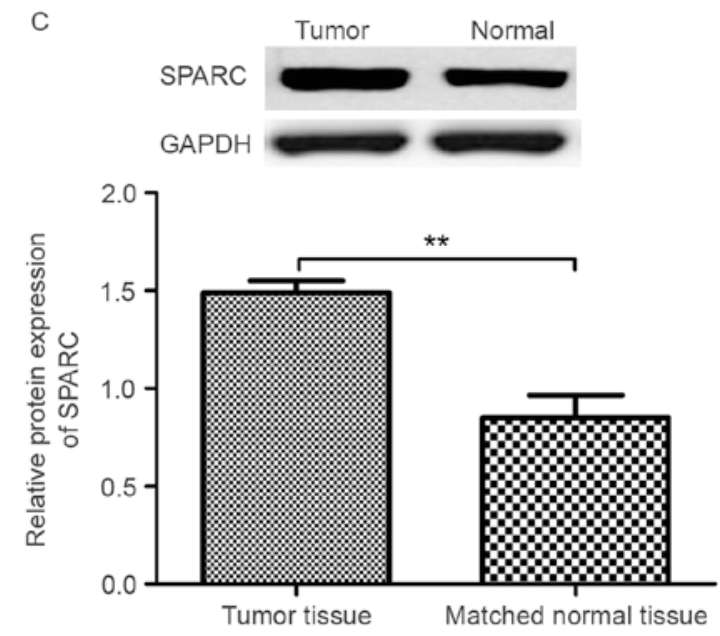

$\mathrm{E}$

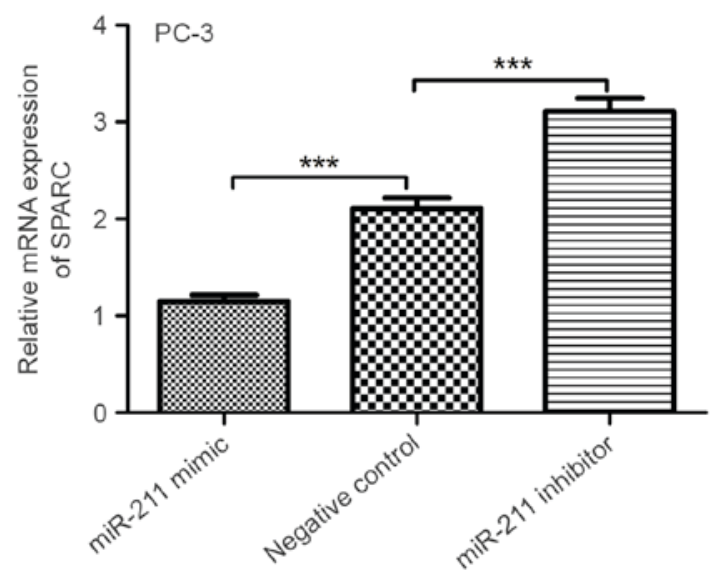

Figure 3. miR-211 regulation of SPARC expression. (A) A computational algorithm revealed that the putative miR-211-binding sequence was in the 3'-UTR of SPARC. (B) RT-qPCR analysis of SPARC mRNA expression in PCa tissues and matched normal prostate tissues. (C) Western blot analysis of SPARC protein expression in PCa tissues and matched normal prostate tissues. (D) RT-qPCR analysis of SPARC expression in the DU145 cells transfected with miR-211 mimics or miR-211 inhibitor or negative control miRNA. The expression of SPARC was normalized to that of GAPDH. (E) RT-qPCR analysis of SPARC expression in the PC-3 cells transfected with miR-211 mimics or miR-211 inhibitor or negative control miRNA. GAPDH was also detected as a loading control. ${ }^{* *} \mathrm{P}<0.01$ and ${ }^{* * *} \mathrm{P}<0.001$. miR-211, microRNA-211; SPARC, secreted protein acidic and rich in cysteine; 3'-UTR, 3'-untranslated region; RT-qPCR, reverse transcription-quantitative polymerase chain reaction; $\mathrm{PCa}$, prostate cancer.

lines. As depicted in Fig. 2B and C, the expression level of miR-211 in the PCa cell lines transfected with miR-211 mimic was significantly higher than those transfected with the negative control miRNA $(\mathrm{P}<0.001)$. Conversely, the miR-211 expression level in the PCa cell lines transfected with the miR-211 inhibitor was significantly lower than those transfected with the negative control miRNA $(\mathrm{P}<0.001)$. The cell proliferation rate in these miRNA-transfected cell lines was also examined. As revealed in Fig. 2D and E, the cell proliferation rate in the PCa cell lines transfected with miRNAs was as follows: PCa cell lines transfected with miR-211 inhibitor, PCa cell lines transfected with negative control miRNA and PCa cell lines transfected with miR-211 mimic.
Expression of SPARC is inversely associated with miR-211. It was revealed that the 3'-untranslated region (3'-UTR) of SPARC contained a conserved putative target site for miR-211 using bioinformatics analysis (Fig. 3A). Therefore, the expression level of SPARC in PCa tumor tissues and matched normal prostate tissue was examined. As revealed in Fig. 3B and C, RT-qPCR and western blot analyses demonstrated that the expression of SPARC in PCa tumor tissues was significantly higher than in matched normal prostate tissue $(\mathrm{P}<0.01)$. The expression level of SPARC in the miR-211 mimic, miR-211 inhibitor and negative control miRNA transfected PCa cell lines was also examined. The SPARC mRNA expression level in the miR-211-mimic-transfected PCa cell lines was significantly lower than in the 


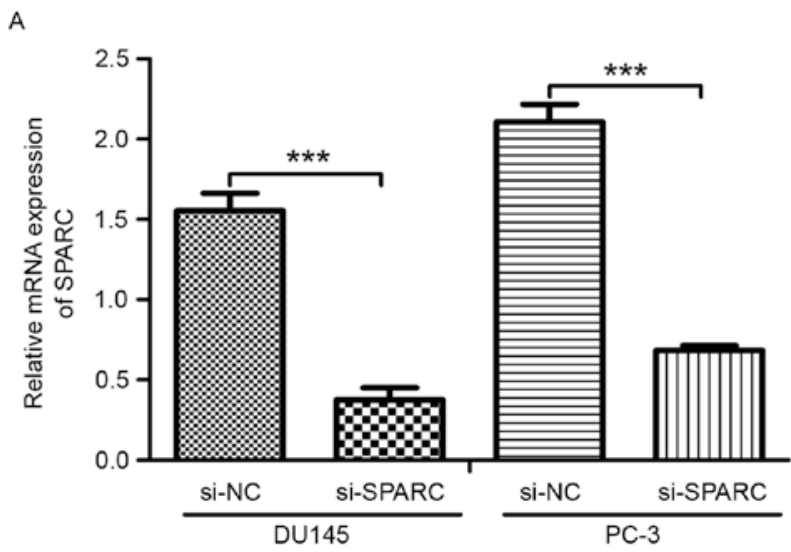

B

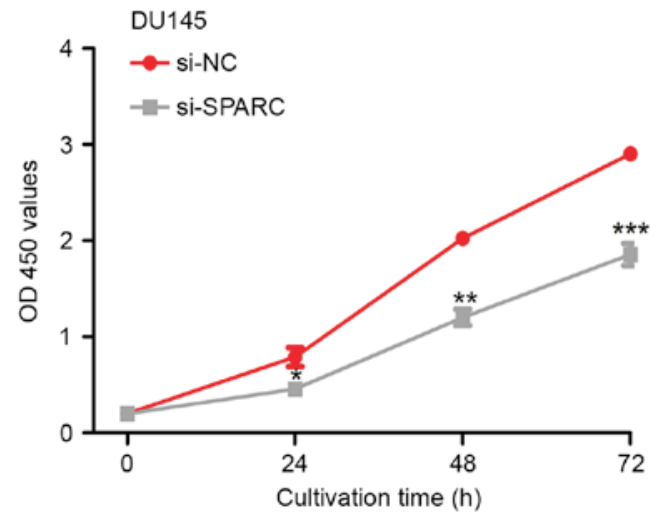

C

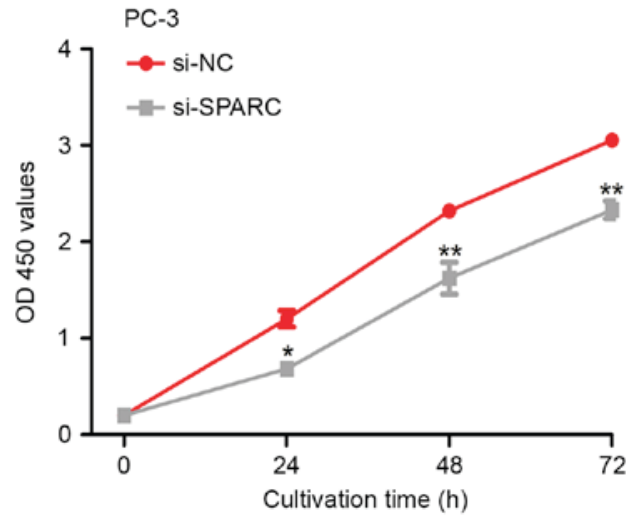

Figure 4. Effect of SPARC on cell proliferation. (A) RT-qPCR analysis of the mRNA level of SPARC in the PCa DU145 and PC-3 cell lines transfected with si-NC or si-SPARC. (B) CCK-8 proliferation assay revealed that knockdown of SPARC by si-SPARC significantly inhibited the proliferation of the DU145 cells compared to the negative control. (C) The CCK-8 proliferation assay revealed that knockdown of SPARC by si-SPARC significantly inhibited the proliferation of the PC-3 cells compared to the negative control. ${ }^{*} \mathrm{P}<0.05,{ }^{* *} \mathrm{P}<0.01$ and ${ }^{* * *} \mathrm{P}<0.001$ vs. si-NC. si-SPARC, small interfering RNA targeted at secreted protein acidic and rich in cysteine; $\mathrm{NC}$, negative control; $\mathrm{PCa}$, prostate cancer; $\mathrm{CCK}-8$, cell counting kit-8; OD, optical density.

miR-211-inhibitor-transfected PCa cell lines (Fig. 3D and E; $\mathrm{P}<0.01)$.

Effect of SPARC on cell proliferation. To determine the role of SPARC on cell proliferation, knockdown of SPARC expression was performed using a specific siRNA in PCa cell lines (DU145 and PC-3). The expression of SPARC transfected with SPARC-siRNA was significantly decreased compared with the negative control (Fig. 4A; $\mathrm{P}<0.001$ ). As expected, knockdown of SPARC inhibited the cell proliferation in PCa cell lines compared with the cells transfected with the negative control (Fig. 4B and C; $\mathrm{P}<0.05$ ).

\section{Discussion}

The established pre-treatment prognostic parameters for patients with PCa currently include Gleason grade, PSA and clinical stage (30-32). Although these parameters are statistically powerful, they are not always sufficient for individual treatment decision optimization (29). It can be hoped that further understanding of PCa biology will lead to improvements in clinical molecular tests that enable the reliable prediction of PCa aggressiveness.

The role of miRNA dysregulation in cancer has been demonstrated by numerous studies $(33,34)$. Previous studies have revealed that miR-211 is aberrantly expressed in number of tumor types and serves an important role in tumor progression (13-18). In the present study, the results of RT-qPCR analysis indicated that the expression of miR-211 was reduced in PCa tissues compared with the matched normal prostate tissues. These results indicated that the dysregulation of miR-211 may be involved in the tumorigenesis of PCa. Furthermore, the association between miR-211 expression and the clinicopathological features was analyzed. It was revealed that the miR-211 expression was significantly associated with tumor stage $(\mathrm{P}=0.035)$ and higher Gleason scores $(\mathrm{P}=0.013)$, indicating that miR-211 expression was associated with the PCa malignancy. It was also revealed that miR-211 expression was reduced in PCa cell lines compared with NHPE cells. Overexpression of miR-211 in PCa cell lines induced by miR-211 mimics significantly inhibited the proliferation of PCa cells; conversely, downregulation of miR-211 using a miR-211 inhibitor promoted the proliferation of PCa cell lines. These results indicated that miR-211 may be a tumor suppressor in the development and progression of PCa.

Previous studies have identified the target genes of miR-211 in a variety of human types of cancer (13-18). To investigate the molecular mechanism whereby miR-211 inhibits the proliferation rate of selected cell lines further, bioinformatics analysis was used to predict the potential downstream target in the PCa 
cell lines. It was revealed that the 3'-UTR of SPARC mRNA contained a complementary sequence for miR-211. The expression of SPARC was increased in PCa tissues compared with matched normal prostate tissues. In the PCa cell lines, it was revealed that the enforced expression of miR-211 could reduce the expression of SPARC. However, the downregulation of miR-211 could increase the expression of SPARC. Therefore, it was assumed that SPARC was a direct target of miR-211 in PCa cell lines.

A previous study has demonstrated that SPARC serves an important role in the development of human hepatocellular carcinoma (16). To investigate the role of SPARC in PCa progression, the expression of SPARC in PCa cell lines was knocked down by siRNA. It was revealed that the proliferation of the PCa cell lines was altered through the downregulation of the expression of SPARC. These results indicated that the knockdown of SPARC elicited similar effects to miR-211 overexpression, and that SPARC is a functionally important target of miR-211.

In conclusion, miR-211 was identified to be frequently downregulated in $\mathrm{PCa}$, which was associated with $\mathrm{PCa}$ progression. Additionally, miR-211 overexpression inhibited cell proliferation in vitro via direct targeting of SPARC. Downregulation of SPARC in a miR-211-mediated manner could shed further light on the molecular mechanisms behind malignancy.

\section{References}

1. Hashimoto Y, Shiina M, Kato T, Yamamura S, Tanaka Y, Majid S, Saini S, Shahryari V, Kulkarni P, Desgupta P, et al: The role of miR-24 as a race related genetic factor in prostate cancer. Oncotarget 8: 16581-16593, 2017.

2. Siegel RL, Miller KD and Jemal A: Cancer statistics, 2016. CA Cancer J Clin 66: 7-30, 2016.

3. Hu J, He J, Kuang Y, Wang Z, Sun Z, Zhu H and Liu X: Expression and significance of $90 \mathrm{~K} / \mathrm{Mac}-2 \mathrm{BP}$ in prostate cancer. Exp Ther Med 5: 181-184, 2013

4. Chen W, Zheng R, Baade P, Zhang S, Zeng H, Bray F, Jemal A, Yu XQ and He J: Cancer statistics in China, 2015. CA Cancer J Clin 66: 115-132, 2016

5. Etzioni R, Urban N, Ramsey S, McIntosh M, Schwartz S, Reid B, Radich J, Anderson G and Hartwell L: The case for early detection. Nat Rev Cancer 3: 243-252, 2003.

6. Bartel DP: MicroRNAs: Target recognition and regulatory functions. Cell 136: 215-233, 2009.

7. Lee RC, Feinbaum RL and Ambros V: The C. Elegans heterochronic gene lin-4 encodes small RNAs with antisense complementarity to lin-14. Cell 75: 843-854, 1993.

8. Chen JF, Mandel EM, Thomson JM, Wu Q, Callis TE, Hammond SM, Conlon FL and Wang DZ: The role of microRNA-1 and microRNA-133 in skeletal muscle proliferation and differentiation. Nat Genet 38: 228-233, 2006.

9. Ambros V: The functions of animal microRNAs. Nature 431: 350-355, 2004

10. Croce CM and Calin GA: miRNAs, cancer, and stem cell division. Cell 122: 6-7, 2005.

11. Ambros V: MicroRNA pathways in flies and worms: Growth, death, fat, stress, and timing. Cell 113: 673-676, 2003.

12. Lu YC, Chang JT, Chan EC, Chao YK, Yeh TS, Chen JS and Cheng AJ: miR-196, an emerging cancer biomarker for digestive tract cancers. J Cancer 7: 650-655, 2016.

13. Lee H, Lee S, Bae H, Kang HS and Kim SJ: Genome-wide identification of target genes for miR-204 and miR-211 identifies their proliferation stimulatory role in breast cancer cells. Sci Rep 6: 25287, 2016.

14. Wang CY, Hua L, Sun J, Yao KH, Chen JT, Zhang JJ and Hu JH: MiR-211 inhibits cell proliferation and invasion of gastric cancer by down-regulating SOX4. Int J Clin Exp Pathol 8: 14013-14020, 2015.
15. Jiang G, Cui Y, Yu X, Wu Z, Ding G and Cao L: miR-211 suppresses hepatocellular carcinoma by downregulating SATB2. Oncotarget 6: 9457-9466, 2015.

16. Deng B, Qu L, Li J, Fang J, Yang S, Cao Z, Mei Z and Sun X: MiRNA-211 suppresses cell proliferation, migration and invasion by targeting SPARC in human hepatocellular carcinoma. Sci Rep 6: 26679, 2016.

17. Xia B, Yang S, Liu T and Lou G: miR-211 suppresses epithelial ovarian cancer proliferation and cell-cycle progression by targeting Cyclin D1 and CDK6. Mol Cancer 14: 57, 2015.

18. Ye L, Wang $\mathrm{H}$ and Liu B: miR-211 promotes non-small cell lung cancer proliferation by targeting SRCIN1. Tumor Biol 37: 1151-1157, 2016.

19. Motamed K: SPARC (osteonectin/BM-40). Int J Biochem Cell Biol 31: 1363-1366, 1999.

20. Shin M, Mizokami A, Kim J, Ofude M, Konaka H, Kadono Y, Kitagawa Y, Miwa S, Kumaki M, Keller ET and Namiki M: Exogenous SPARC suppresses proliferation and migration of prostate cancer by interacting with integrin $\beta 1$. Prostate 73 : 1159-1170, 2013

21. Shi Q, Bao S, Maxwell JA, Reese ED, Friedman HS, Bigner DD, Wang XF and Rich JN: Secreted protein acidic, rich in cysteine (SPARC), mediates cellular survival of gliomas through AKT activation. J Biol Chem 279: 52200-52209, 2004.

22. Thomas R, True LD, Bassuk JA, Lange PH and Vessella RL: Differential expression of osteonectin/SPARC during human prostate cancer progression. Clin Cancer Res 6: 1140-1149, 2000.

23. Zhao ZS, Wang YY, Chu YQ, Ye ZY and Tao HQ: SPARC is associated with gastric cancer progression and poor survival of patients. Clin Cancer Res 16: 260-268, 2010.

24. Massi D, Franchi A, Borgognoni L, Reali UM and Santucci M: Osteonectin expression correlates with clinical outcome in thin cutaneous malignant melanomas. Hum Pathol 30: 339-344, 1999.

25. Sharma S, Xing F, Liu Y, Wu K, Said N, Pochampally R, Shizawa Y, Lin HK, Balaji KC and Watabe K: Secreted Protein Acidic and Rich in Cysteine (SPARC) mediates metastatic dormancy of prostate cancer in bone. J Biol Chem 291: 19351-19363, 2016.

26. Edge SB and Compton CC: The American Joint Committee on Cancer: The 7th edition of the AJCC cancer staging manual and the future of TNM. Ann Surg Oncol 17: 1471-1474, 2010.

27. Livak KJ and Schmittgen TD: Analysis of relative gene expression data using real-time quantitative PCR and the 2(-Delta Delta C(T)) method. Methods 25: 402-408, 2001.

28. Lewis BP, Burge CB and Bartel DP: Conserved seed pairing, often flanked by adensines, indicates that thousands of human genes are microRNA targets. Cell 120: 15-20, 2005.

29. Rusthoven CG, Carlson JA, Waxweiler TV, Yeh N, Raben D, Flaig TW and Kavanagh BD: The prognostic significance of Gleason scores in metastatic prostate cancer. Urol Oncol 32: 707-713, 2014.

30. Cuzick J, Yang ZH, Fisher G, Tikishvili E, Stone S, Lanchbury JS, Camacho N, Merson S, Brewer D, Cooper CS, et al: Prognostic value of PTEN loss in men with conservatively managed localized prostate cancer. Br J Cancer 108: 2582-2589, 2013.

31. Noh BJ, Sung JY, Kim YW, Chang SG and Park YK: Prognostic value of ERG, PTEN, CRISPR 3 and SPINK1 in predicting biochemical recurrence in prostate cancer. Oncol Lett 11: 3621-3630, 2016

32. Duan K, Ge YC, Zhang XP, Wu SY, Feng JS, Chen SL, Zhang LI, Yuan $\mathrm{ZH}$ and $\mathrm{Fu} \mathrm{CH}$ : miR-34a inhibits cell proliferation in prostate cancer by downregulating of SIRT1 expression. Oncol Lett 10: 3223-3227, 2015.

33. Bier A, Giladi N, Kronfeld N, Lee HK, Cazacu S, Finniss S, Xiang C, Poisson L, deCarvalho AC, Slavin S, et al: MicroRNA-137 is downregulated in glioblastoma and inhibits the stemness of glioma stem cells by targeting RTVP-1. Oncotarget 4: 665-676, 2013.

34. Lu C, Liao Z, Cai M and Zhang G: MicroRNA-320a downregulation mediates human liver cancer cell proliferation through the Wnt/ $\beta$-catenin signaling pathway. Oncol Lett 13: 573-578, 2017.

This work is licensed under a Creative Commons Attribution-NonCommercial-NoDerivatives 4.0 International (CC BY-NC-ND 4.0) License. 